\title{
Desplazamiento de Tercer Molar Superior a Fosa Infratemporal: Reporte de Casos y Revisión de Literatura
}

\author{
Maxillary Third Molar Displacement into the Infratemporal Fossa: \\ Case Report and Literature Review
}

\author{
Perla María Barrera Posada1; Daniel Alejandro Guerra Leal² \& Jorge Alberto Martínez Treviño³
}

BARRERA, P. P. M.; GUERRA, L. D. A. \& MARTíNEZ, T. J. A. Desplazamiento de tercer molar superior a fosa infratemporal: reporte de casos y revisión de literatura. Int. J. Odontostomat., 15(4):827-834, 2021.

RESUMEN: Dentro de las complicaciones transoperatorias de una cirugía de tercer molar se encuentra el desplazamiento del diente a espacios anatómicos adyacentes. Dicho accidente representa un porcentaje muy bajo de las complicaciones y se atribuye a una planeación y manejo clínico inadecuado. En este reporte se abordará específicamente el desplazamiento accidental de terceros molares superiores a la fosa infratemporal. Se realizó una búsqueda de información en la plataforma PubMed con las palabras claves "third molar displacement", obteniendo un total de 22 artículos según los criterios de inclusión. Se presentan dos casos de desplazamiento de tercer molar superior a fosa infratemporal manejados en el Centro Académico de Atención Odontológica (CAAD) del Tecnológico de Monterrey. El propósito del presente escrito es proponer un protocolo de manejo para dicha complicación.

PALABRAS CLAVE: tercer molar, fosa infratemporal, complicaciones transoperatorias.

\section{INTRODUCCIÓN}

La fosa infratemporal es un espacio localizado en la intersección de importantes estructuras anatómicas como la arteria maxilar, el plexo venoso pterigoideo, el ganglio ótico, la cuerda del tímpano, las ramas del nervio mandibular y esfenopalatino, así como los músculos pterigoideo lateral y medial (Fig. 1) (Di Nardo et al., 2019).

El desplazamiento de terceros molares superiores hacia la fosa infratemporal se considera una complicación transoperatoria rara, pero delicada, de la cirugía de terceros molares. Su incidencia se desconoce ya que es reportada en un muy bajo porcentaje en comparación con las otras complicaciones.

El primer caso reportado fue publicado en 1977 por Winkler, donde se reportó un desplazamiento de tercer molar superior hacia la fosa infratemporal con síntomas como limitación a la apertura y diplopía. Clínicamente se manejó con un acceso transinusal inmediato, realizando una ventana ósea en la pared posterior del seno maxilar para recuperar el diente. Posteriormente, se refiere recuperación sin complicaciones, apertura oral normal con ligera persistencia de la diplopía (Winkler et al., 1977).

Durante una cirugía de terceros molares, el diente puede llegar a ser desplazado accidentalmente hacia espacios anatómicos adyacentes por diversos motivos. En el caso de los terceros molares superiores, se han reportado desplazamientos hacia la fosa infratemporal, fosa pterigopalatina, espacio oral y seno maxilar. En el caso de los terceros molares inferiores se han reportado desplazamientos hacia el espacio submandibular, espacio pterigomandibular, espacio sublingual y espacio faríngeo lateral (Lutz et al., 2019). En este reporte se aborda el desplazamiento accidental de terceros molares superiores a la fosa infratemporal y se analizan dos casos con su respectivo manejo clínico.

\footnotetext{
${ }^{1}$ Escuela de medicina y ciencias de la salud, Tec Salud, Monterrey, México.

${ }^{2}$ Coordinador del Centro Académico de Atención Odontológica, Escuela de medicina y ciencias de la salud, Tec Salud, Monterrey, México.

${ }^{3}$ Director Asociado de División Ciencias de la Salud, Escuela de Medicina y Ciencias de la Salud, Tec Salud, Monterrey, México.
} 


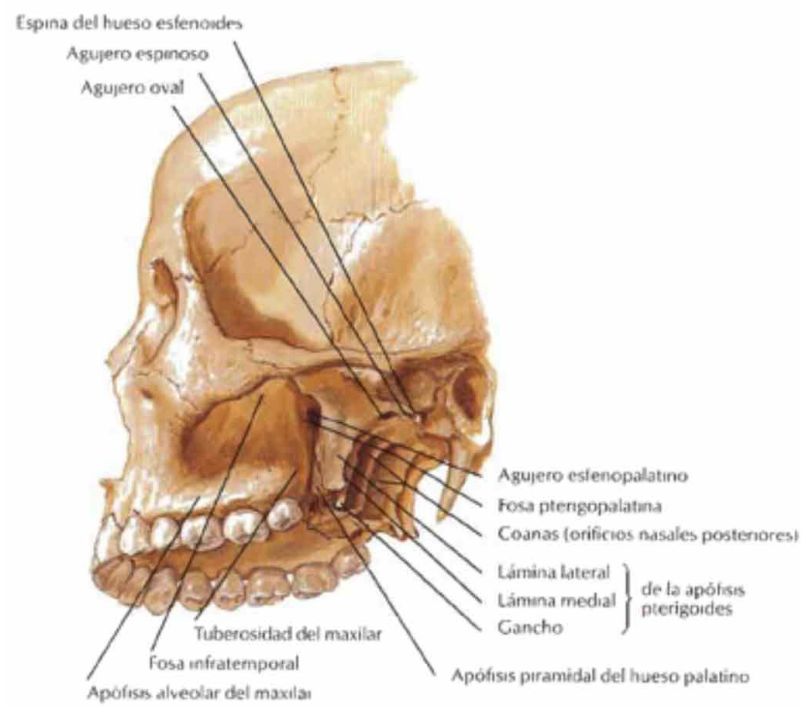

Fig. 1. Imágenes tomadas del Atlas de Anatomía Humana (Netter, 2007).

Como se mencionó, un desplazamiento dental hacia un espacio anatómico como resultado de una complicación, no es reportado con frecuencia (Sencimen et al., 2017). Sin embargo, según los casos que han sido publicados, dicho accidente se puede atribuir a diversas causas provenientes de un inadecuado manejo clínico. Dentro de las principales causas encontramos una técnica o diseño de abordaje inapropiado, el uso excesivo de fuerza, la falta de visibilidad, el uso incorrecto del instrumental, la falta de experiencia o un manejo apresurado y un exceso de confianza del operador (Bouloux et al., 2007; Shahakbari et al., 2011; Selvi et al., 2011; Sencimen et al.). Estos factores contribuyen a incrementar el riesgo de presentar un desplazamiento dental, dejando al diente fuera del campo de visibilidad y alcance del operador.

Otro factor importante a considerar es la localización anatómica de los terceros molares superiores, los cuales frecuentemente se encuentran impactados con un patrón de erupción en sentido distal, y este mismo se continua al realizar su luxación y posterior elevación, lo que hace posible que este diente sea más susceptible a ser desplazado a la fosa infratemporal (Martínez, 2009).

\section{MATERIAL Y MÉTODO}

La revisión de literatura se realizó bajo la búsqueda de "third molar displacement" en la base de datos de PubMed. Se incluyeron artículos desde 1977

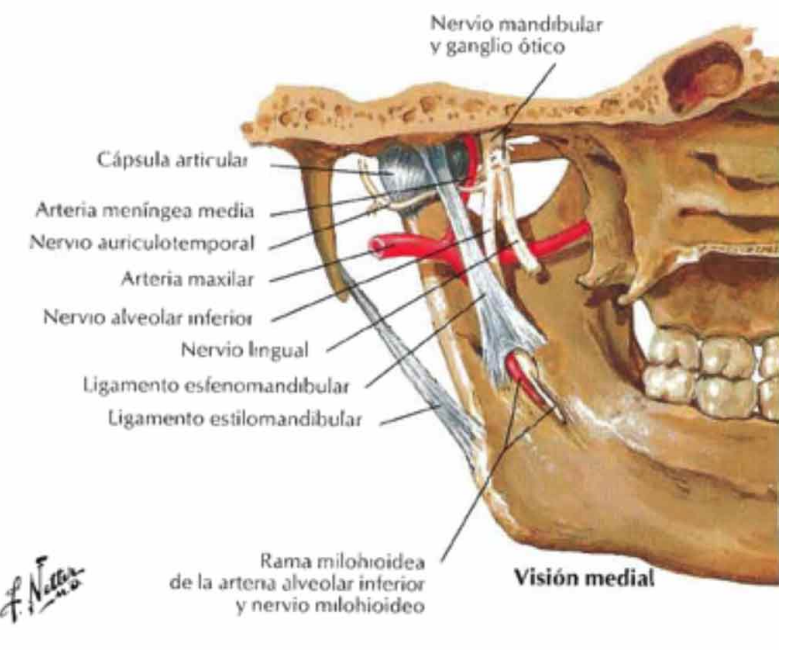

hasta el 2020 relacionados con el desplazamiento de tercer molar superior a la fosa infratemporal. Se excluyeron artículos sobre desplazamiento de dientes a diferentes espacios anatómicos. Como resultado de la búsqueda se obtuvieron un total de 22 artículos reportando casos de desplazamiento de tercer molar superior a fosa infratemporal. Estos se enlistan según su técnica, síntomas y resultados en la Tabla I.

\section{REPORTE DE CASOS}

Caso 1. Paciente femenina de 29 años de edad, la cual es referida al departamento de Cirugía Maxilofacial del Centro Académico de Atención Odontológica (CAAD) del Tecnológico de Monterrey, por presentar desplazamiento de tercer molar superior a tejidos blandos de 24 horas de evolución. Durante la consulta refiere dolor moderado, clínicamente presenta ligero edema facial que se acompaña de restricción de movimientos mandibulares. Se toma radiografía panorámica en la que se observa al diente desplazado en dirección posterior, posiblemente hacia la fosa infratemporal (Fig. 2A). Se decide manejar con terapia antimicrobiana, analgésica y antiinflamatoria para proteger y disminuir sintomatología durante etapa aguda. Se mantiene en observación realizando controles semanales para normar conducta a seguir.

Posterior a las 3 semanas del accidente, se propone realizar remoción quirúrgica del diente desplazado bajo anestesia local infiltrativa a base de lidocaína $2 \%$ con epinefrina 1:100,000. Se procede a realizar 
BARRERA, P. P. M.; GUERRA, L. D. A. \& MARTínEZ, T. J. A. Desplazamiento de tercer molar superior a fosa infratemporal: reporte de casos y revisión de literatura. Int. J. Odontostomat., 15(4):827-834, 2021

Tabla I. Resumen cronológico de los casos publicados de desplazamiento de tercer molar superior a la fosa infratemporal.

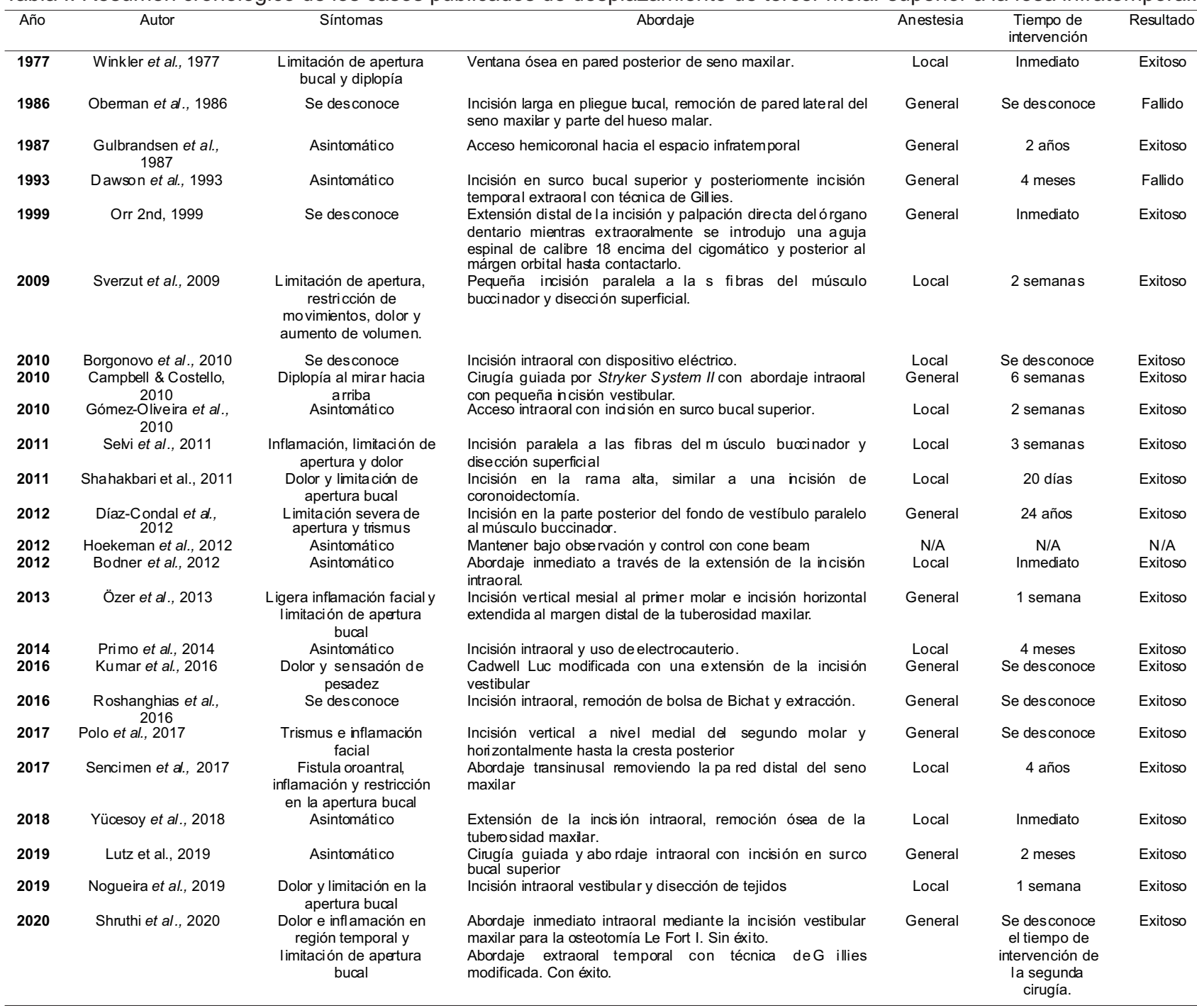
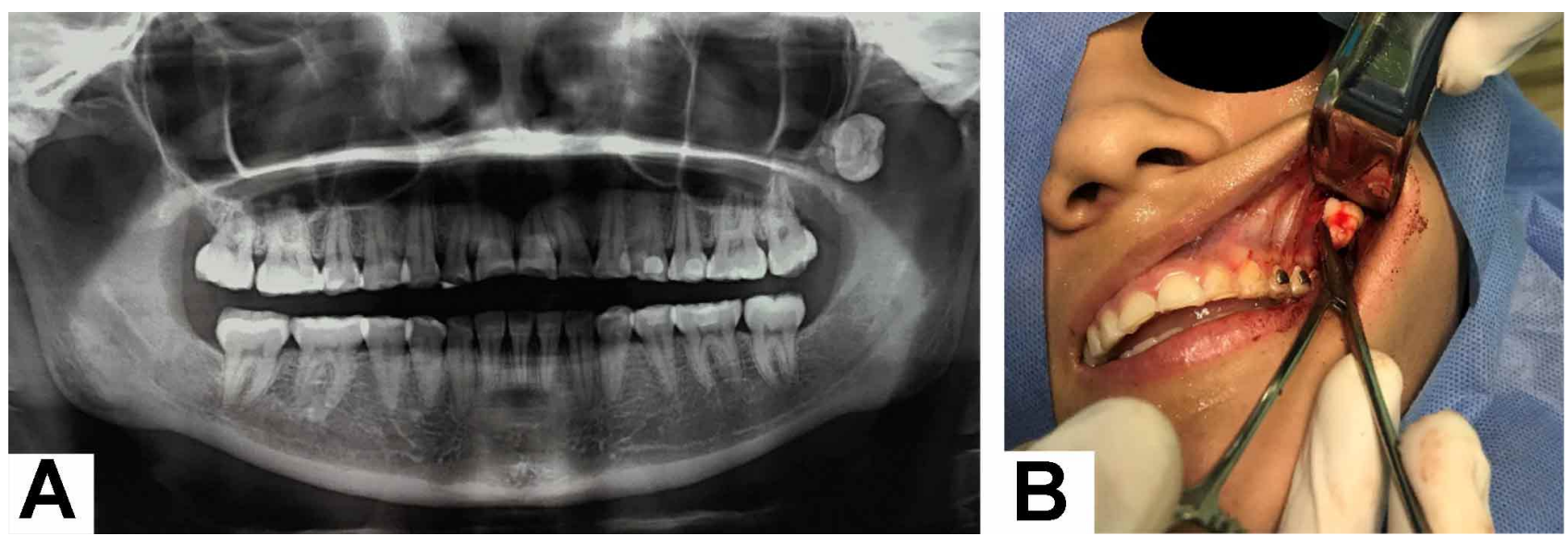

Fig. 2. (A) Radiografía panorámica mostrando el desplazamiento del tercer molar superior izquierdo en dirección posterior, posiblemente hacia la fosa infratemporal. (B). Localización y retiro del diente. 
incisión en fondo de vestíbulo de $2 \mathrm{~cm}$ de longitud, se comienza disección roma con pinzas mosco curvas y se retrae tejido con separadores Farabeuf, se localiza diente, teniendo precaución con las estructuras vasculares de la región. Una vez que se localiza, se sujeta firmemente con pinzas de Allis, para su retiro. Se lava herida con solución fisiológica y se procede a realizar reparación de la herida con catgut simple 4-0 y puntos discontinuos (Fig. 2B). Se dan indicaciones postquirúrgicas de cuidados e higiene. Se indica antibiótico terapia a base de Cefalexina 500 mg VO cada 8 horas, durante 7 días y analgesia con Ketorolaco 10 mg VO cada 6 horas para el manejo del dolor. Se cita a los 5 y 14 días para su control postoperatorio, observando una evolución favorable.

Caso 2. Paciente femenina de 18 años de edad, la cual refiere que durante la luxación del tercer molar superior derecho, este se salió del campo de visibilidad al desplazarse posteriormente hacia los tejidos blandos adyacentes. Se tomó radiografía panorámica
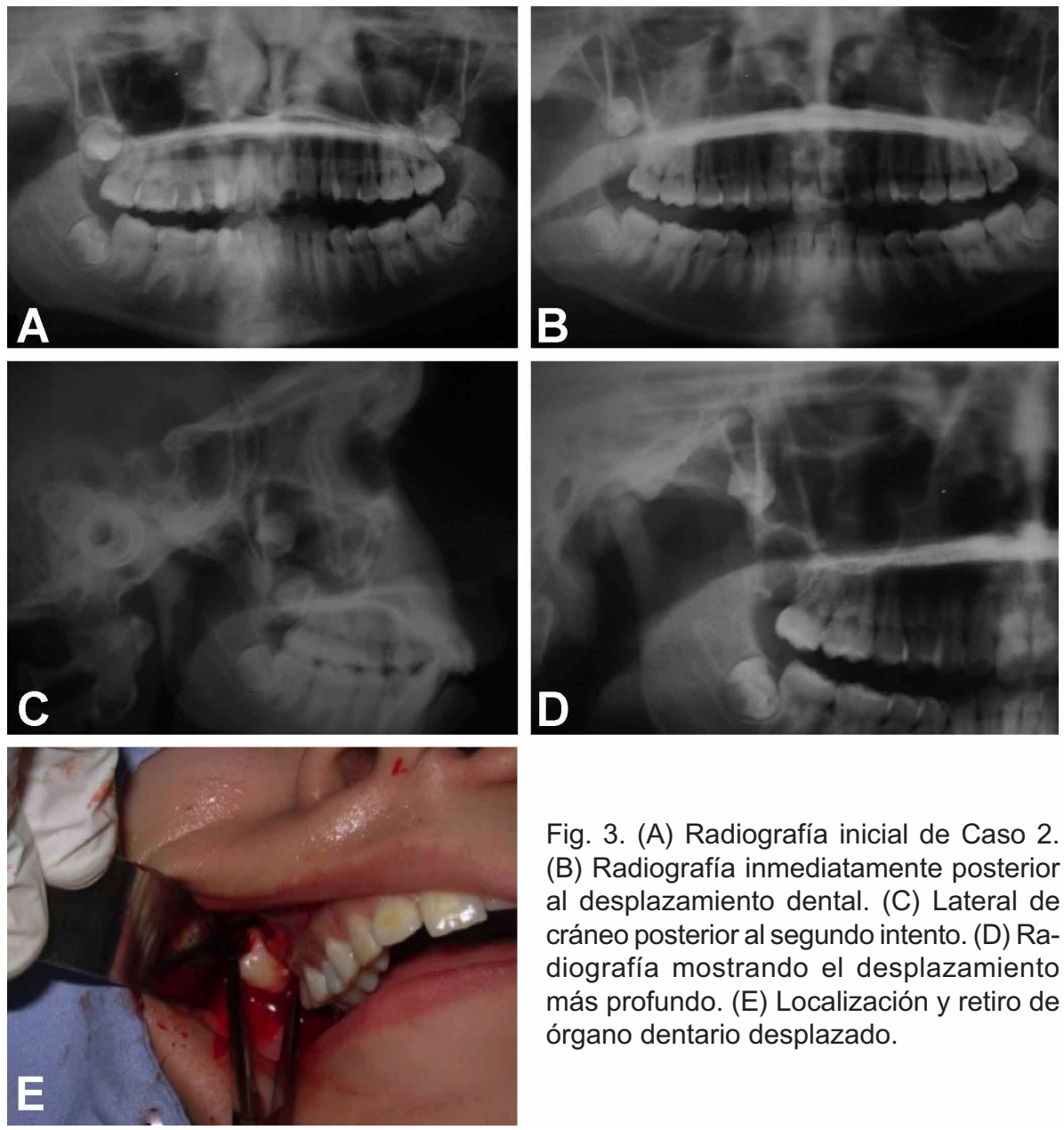

Fig. 3. (A) Radiografía inicial de Caso 2. (B) Radiografía inmediatamente posterior al desplazamiento dental. (C) Lateral de cráneo posterior al segundo intento. (D) Radiografía mostrando el desplazamiento más profundo. (E) Localización y retiro de órgano dentario desplazado. inmediatamente para localizarlo (Fig. 3B) y se decidió extender incisión vestibular para intentar extraerlo. Sin embargo, no se obtuvo éxito alguno e incluso se provocó un desplazamiento más profundo. Se decide volver a tomar radiografía panorámica (Fig. 3C) y una radiografía lateral de cráneo (Fig. 3D).

Posteriormente se decide referir al departamento de cirugía maxilofacial del CAAD. A la exploración intraoral no se logra palpar al diente entre el tejido blando. La paciente se presenta aparentemente asintomática, por lo que se indica comenzar con terapia antimicrobiana, analgésica y antiinflamatoria para proteger y disminuir sintomatología durante la etapa aguda. Se propone realizar remoción quirúrgica del diente desplazado posterior a 3 semanas de incidente y mantener controles semanales.

Cumplida la tercer semana se decide realizar procedimiento para recuperar el diente bajo anestesia local infiltrativa. Se realiza incisión lo más conservadora posible en fondo de vestíbulo de $2 \mathrm{~cm}$ de longitud y se realiza disección roma con pinzas mosco curvas. Se retrae tejido con separadores Farabeuf y se diseca hasta localizar al tercer molar desplazado. Una vez que se sujeta firmemente, se retira. Se lava la herida con solución fisiológica y se sutura (Fig. 3E). Se dan indicaciones post quirúrgicas de cuidados e higiene. Se indica Cefalexina 500mg VO cada 8 horas durante 7 días, y Ketorolaco $10 \mathrm{mg}$ VO cada 6 horas para el manejo del dolor. Se cita a los 5 y 14 días para su control postoperatorio, observando una evolución favorable y sin complicaciones. 


\section{DISCUSIÓN}

La remoción quirúrgica de un diente ubicado en la fosa infratemporal representa un nivel de complejidad elevado debido a su localización y las posibles complicaciones clínicas a presentar. Cuando un tercer molar superior se desplaza hacia la fosa infratemporal, generalmente se realiza a través del periostio y se ubica lateral a la cara infratemporal del ala mayor del esfenoides e inferior al músculo pterigoideo lateral (Abubaker et al., 2016).

Como ya se mencionó, la fosa infratemporal se encuentra en cercanía con estructuras anatómicas vitales como la arteria maxilar, el plexo venoso pterigoideo, las ramas de los nervios mandibular y esfeno palatino, por lo tanto, la remoción del diente desplazado representa riesgos potenciales, tales como hemorragias, daños neurológicos o incluso una futura migración coronal hacia la base del cráneo (Selvi et al.; Özer et al., 2013; Lutz et al.). Asimismo, existe una alta probabilidad de presentar complicaciones secundarias al desplazamiento del tercer molar superior a fosa infratemporal, como infección, limitación en la apertura oral, inflamación, diplopía, dolor, reacción por cuerpo extraño y trismus. Estas son reportadas con frecuencia en los casos publicados de terceros molares desplazados a la fosa infratemporal (Selvi et al.; Di Nardo et al.; Lutz et al.).

La aparición de la diplopía reportada como complicación transitoria en los casos de desplazamiento del tercer molar superior a la fosa infratemporal, puede ser atribuida a la infiltración del anestésico local. Se han sugerido varios mecanismos anatómicos para explicar cómo el agente anestésico podría acceder a las estructuras necesarias para producir una oftalmoplejía (Ceylan et al., 2010). Se han reportado diferentes vías vasculares, neurológicas, miofasciales y redes linfáticas como rutas potenciales por las cuales la solución de anestésico local puede llegar hasta el área de la órbita afectando así los músculos del bulbo ocular y dando como resultado la diplopía (Boynes et al., 2010).

La opción de no retirar el tercer molar desplazado a fosa infratemporal pudiera llegar a ser elegida por el paciente, principalmente si éste se encuentra asintomático al momento de la consulta. En la literatura, esta opción ha sido propuesta, sin embargo, se refiere que la mayoría de los pacientes regresan a consulta debido a la aparición de síntomas. Aunque cabe mencionar, que gran número de los reportes publica- dos abordan casos con pacientes sintomáticos, por lo cual, se documenta la remoción del tercer molar desplazado a fosa infratemporal (Sencimen et al.). Por consiguiente, se debe informar al paciente detalladamente la serie de complicaciones secundarias y síntomas posibles a aparecer aun cuando estas pudieran no presentarse por un largo periodo. De esta manera, el paciente puede evaluar el riesgo-beneficio de retirar o no el tercer molar desplazado.

El tiempo adecuado para realizar la intervención quirúrgica posterior al desplazamiento dental, permanece controversial en la literatura (Sverzut et al., 2009; Selvi et al.; Özer et al.), ya que algunos autores sugieren la remoción lo antes posible para evitar el desarrollo de complicaciones y aparición de síntomas (Selvi et al.; Özer et al.). Estos aseguran que al incorporar el uso del cone beam, es posible la remoción inmediata del tercer molar basada en las imágenes precisas que se obtienen (Bodner et al., 2012; Yücesoy et al., 2018). Por otro lado, se han reportado casos de intervenciones exitosas con rangos de entre 1 y 4 años posteriores al desplazamiento accidental, aunque en la mayoría de los casos, se opta por realizar el procedimiento antes del primer mes (Lutz et al.).

De acuerdo a la literatura, el postergar la extracción permite que se conforme una fibrosis alrededor del diente, lo cual lo estabilizará y facilitará su localización y posterior extracción (Sverzut et al.; Primo et al., 2014). De igual forma, algunos autores han reportado la migración espontánea del diente hacia la cavidad oral debido a los movimientos masticatorios naturales (Gómez-Oliveira et al., 2010; Özer et al.), ya que durante la masticación, los músculos pterigoideos lateral y medial, localizados en los márgenes de la fosa infratemporal, se contraen y relajan periódicamente. El proceso coronoides se mantiene en movimiento por la acción del músculo temporal y en conjunto con la gravedad, son factores contribuyentes para que gradualmente el diente desplazado pueda migrar (Selvi et al.).

Dentro de la literatura se pueden encontrar diversos artículos sobre terceros molares desplazados hacia la fosa infratemporal con diferentes manejos, abordajes y tiempos de intervención. El primer caso, como ya se mencionó, fue reportado en 1977 (Winkler et al.). Posteriormente se han publicado casos con diferentes abordajes quirúrgicos como incisiones en el surco bucal, técnica de Gillies, de Cadwell- Luc, a través del seno maxilar o abordajes más conservadores (Sverzut et al.; Selvi et al.; Özer et al.). 
El desplazamiento accidental es asociado a una planeación y ejecución inapropiada, de tal manera, la prevención se basa en un correcto diagnóstico, valoración clínica y radiográfica, selección adecuada de instrumentos y técnica a emplear. Si durante el procedimiento se sospecha que el diente puede desplazarse, se aconseja extender la incisión para ampliar el campo de trabajo. Si esta complicación llega a ocurrir, inmediatamente se debe de detener el procedimiento, referir al paciente al cirujano maxilofacial y no intentar remover el diente (Özer et al.; Primo et al.).

Protocolo. De acuerdo a la revisión de la literatura, experiencia y casos clínicos presentados, se pretende establecer un protocolo de atención para disminuir el riesgo del desplazamiento de terceros molares superiores a la fosa infratemporal, así como una guía en caso de presentar la complicación.

El enfoque actual de cualquier rama del área de la salud está centrado en la prevención, ya que el mejor manejo de cualquier complicación es evitarla. Con dicha finalidad se proponen cinco principios indispensables para llevar a cabo una buena practicar quirúrgica.

1. Radiografía. Las radiografías forman parte de los métodos auxiliares de diagnóstico, los cuales contribuyen a lograr un diagnóstico acertado y posteriormente a establecer el plan de tratamiento idóneo. Ya sea una radiografía panorámica o una periapical, debe permitir evaluar el diente completo y las estructuras inmediatas a este. Con base en una radiografía se puede estimar el grado de complejidad del tratamiento y por ende, determinar si el operador es competente en realizar el procedimiento, según sus propias limitaciones.

2. Anestesia. El dominio de los anestésicos locales es fundamental para desarrollar una buena práctica odontológica. Siempre se debe de corroborar que la anestesia local haya sido satisfactoria antes de iniciar, ya que una buena técnica de anestesia local permite que el procedimiento sea mucho más eficaz y tranquilo, tanto para el operador como para el paciente, evitando movimientos bruscos involuntarios del paciente a causa de dolor. No es necesario trabajar bajo anestesia general o sedación, si el procedimiento o el paciente no lo ameritan.

3. Instrumental. Antes de iniciar cualquier tratamiento quirúrgico se debe contar con el equipo adecuado para llevarlo a cabo. Esto incluye el instrumental necesario no sólo para el procedimiento, sino también para resolver las posibles complicaciones derivadas del mismo.
De no ser así el procedimiento no se debe de realizar.

4. Visibilidad. Tener un buen campo de visibilidad del lecho quirúrgico engloba una buena iluminación, ayuda eficiente por parte del asistente, aspiración por succión y un abordaje adecuado. Estos cuatro puntos van de la mano para facilitar el procedimiento, de otra manera la visibilidad se ve obstruida y las probabilidades de presentar una complicación aumenta.

5. Incisión y colgajo. Se debe realizar una adecuada planeación de un colgajo mucoperióstico para el acceso quirúrgico de los dientes. La incisión debe de cumplir ciertos requisitos para evitar complicaciones. Debe ser nítida, tan amplia como sea necesaria pero lo más conservadora posible, de una sola intención, proveer su propio riego sanguíneo, incluir el periostio, ser capaz de reposicionarse en su lugar original, cicatrizar por primera intención, sin tensión y de una amplitud, forma y dirección necesarias para obtener completa visibilidad.

6. Fuerzas. La inadecuada manipulación del diente representa un riesgo importante para desencadenar una complicación. Es imprescindible evitar fuerzas excesivas o ejercerlas en la dirección incorrecta, además, el riesgo aumenta si el instrumental no está posicionado adecuadamente. Por ejemplo, si el separador Minnesota no se encuentra distal al tercer molar superior bloqueando su patrón distal a seguir, al realizar su luxación y elevación, se facilita que el diente sea desplazado.

En el caso de que se presente un desplazamiento dental a la fosa infratemporal se pretende establecer un protocolo a seguir para normar una serie de pautas indispensables con el fin de recuperar el diente y evitar futuras complicaciones.

Tomando en cuenta los conceptos presentados, el desplazamiento del diente a la fosa infratemporal representa riesgos importantes en la salud del paciente. Por lo tanto, una vez que sucede el accidente, se recomienda detener el procedimiento inmediatamente, informar y explicar al paciente sobre el acontecimiento y referirlo al área de cirugía maxilofacial. Una vez que el cirujano maxilofacial recibe al paciente se debe de valorar su estado actual y tomar en cuenta los siguientes puntos para planear la recuperación del diente.

- Información. Una vez presentado el desplazamiento del tercer molar a la fosa infratemporal es de suma importancia informar al paciente sobre lo ocurrido, así como los posibles síntomas a presentar. Esta com- 
plicación puede ocasionar riesgos secundarios, los cuales deben ser explicados claramente ante el paciente para poder plantear un plan de tratamiento.

- Imagenología. Actualmente el avance tecnológico hace posible el acceso a la Tomografía Computarizada (TC), donde se podrá obtener una imagen tridimensional precisa del diente desplazado y sus estructuras adyacentes. Por lo tanto, si se encuentra dentro de las posibilidades, se recomienda indicar un cone beam como auxiliar de diagnóstico. Las radiografías panorámicas, frontales y laterales de cráneo también arrojan información referente al diente desplazado y deberán ser indicadas en caso de no tener una TC.

- Farmacoterapia. Inmediatamente posterior al accidente se recomienda comenzar con un esquema antimicrobiano y analgésico para disminuir el riesgo de una infección y la sintomatología durante la etapa aguda. Este esquema se debe de mantener hasta retirar el diente desplazado. Posterior a la cirugía de recuperación se debe indicar continuar con antibiótico, antiinflamatorio y analgésico con citas de control para monitorear la evolución.

- Tiempo de intervención: No se recomienda intentar recuperar el diente desplazado al momento del accidente, principalmente si el operador no se encuentra capacitado para ello ya que se corre el riesgo de agravar la situación. Una vez que se deriva el paciente y se dan indicaciones, se considera esencial marcar un periodo de espera y control de al menos tres semanas posterior al incidente para realizar la cirugía de recuperación. El tiempo de espera permite la formación de una fibrosis alrededor del diente, lo cual facilitará su manipulación.

- Abordaje. Se considera que la recuperación del diente desplazado a la fosa infratemporal se puede realizar perfectamente con un abordaje intraoral. Se recomienda realizar una incisión en fondo de vestíbulo aproximadamente de $2 \mathrm{~cm}$ de longitud considerando que sea tan amplia como sea necesaria pero lo más conservadora posible.

\section{CONCLUSIONES}

Los accidentes transoperatorios pueden ocurrir durante cualquier cirugía, por lo que las posibles complicaciones ligadas al procedimiento a realizar deben ser consideradas antes de la planeación, ya que el mejor manejo de cualquier complicación es la prevención.
El desplazamiento del tercer molar a la fosa infratemporal es una complicación rara pero posible. La complejidad de enfrentar estos accidentes se debe a la vulnerabilidad de las estructuras anatómicas adyacentes. Seguir las pautas propuestas para realizar un procedimiento quirúrgico reducirá los riesgos de presentar una complicación transoperatoria. Si se llega a presentar una complicación transoperatoria, se aconseja no arriesgarse a intentar realizar trabajos quirúrgicos, tales como la remoción de un tercer molar desplazado, si no se está calificado. Se recomienda que dicha complicación sea derivada y resuelta por un cirujano maxilofacial con experiencia, quien deberá seguir un plan específico basado en el correcto diagnóstico.

BARRERA, P. P. M.; GUERRA, L. D. A. \& MARTínEZ, T. J. A. Maxillary third molar displacement into the infratemporal fossa: case report and literature review. Int. J. Odontostomat.,15(4):827-834, 2021.

ABSTRACT: One of the intraoperative complications of third molar surgery is the displacement of the tooth to adjacent anatomical spaces. This accident represents a small percentage of complications and is attributed to inadequate clinical planning and management. This report will specifically address the accidental displacement of upper third molars to the infratemporal fossa. A search was conducted by using the PubMed database with the keywords "third molar displacement", a total of 22 articles were included according to the inclusion criteria. Two cases of displacement of the upper third molar to the infratemporal fossa managed at the Centro Academico de Atencion Odontologica (CAAD) of the Tecnologico de Monterrey will be reported. The aim of this paper is to present a management protocol for this complication.

KEY WORDS: third molar, infratemporal fossa, intraoperative complications.

\section{REFERENCIAS BIBLIOGRÁFICAS}

Abubaker, A. O.; Lam, D. \& Benson, K. J. Oral and Maxillofacial Surgery Secrets. 3rd ed. St. Louis, Elsevier, 2016. pp.268.

Bodner, L.; Joshua, B. Z. \& Puterman, M. B. Removal of a maxillary third molar from the infratemporal fossa. J. Med. Cases, 3(2):979, 2012.

Borgonovo, A.; Grossi, G. B.; Maiorana, C.; Santoro, F. \& Cicciù, M. Management of the Upper Third Molar Displaced in the Infratemporal Fossa: A Case Report. Int. J. Clin. Dent., 3(4):3018, 2010.

Bouloux, G. F.; Steed, M. B. \& Perciaccante, V. J. Complications of third molar surgery. Oral Maxillofac. Surg. Clin. North Am., 19(1):117-28, 2007.

Boynes, S. G.; Echeverria, Z. \& Abdulwahab, M. Ocular complications associated with local anesthesia administration in dentistry. Dent. Clin. North Am., 54(4):677-86, 2010. 
Campbell, A. \& Costello, B. J. Retrieval of a displaced third molar using navigation and active image guidance. J. Oral Maxillofac. Surg., 68(2):480-5, 2010.

Ceylan, O. M.; Mutlu, F. M. \& Altinsoy, H. I. Transient binocular diplopia as a rare complication of local anesthesia. J. Pediatr. Ophthalmol. Strabismus, 47(6):381-2, 2010.

Dawson, K.; MacMillan, A. \& Wiesenfeld, D. Removal of a maxillary third molar from the infratemporal fossa by a temporal approach and the aid of image-intensifying cineradiography. J. Oral Maxillofac. Surg., 51(12):1395-7, 1993.

Di Nardo, D.; Mazzucchi, G.; Lollobrigida, M.; Passariello, C.; Guarnieri, R.; Galli, M.; De Biase, A. \& Testarelli, L. Immediate or delayed retrieval of the displaced third molar: A review. J. Clin. Exp. Dent., 1;11(1):e55-e61, 2019.

Díaz-Condal, C.; Sánchez, R. R.; García, C. G. \& MuñozSmitmans, C. Eliminación quirúrgica de tercer molar maxilar desde la fosa infratemporal, por trismus severo. Acta Odontol. Venez., 50(3), 2012. Disponible en: https:// www.actaodontologica.com/ediciones/2012/3/art-13/

Gómez-Oliveira, G.; Arribas-García, I.; Alvarez-Flores, M.; Gregoire-Ferriol, J. \& Martínez-Gimeno, C. Delayed removal of a maxillary third molar from the infratemporal fossa. Med. Oral Patol. Oral Cir. Bucal, 15:e509-11, 2010.

Gulbrandsen, S. R.; Jackson, I. T. \& Turlington, E. G. Recovery of a maxillary third molar from the infratemporal space via a hemicoronal approach. J. Oral Maxillofac. Surg., 45(3):279$82,1987$.

Hoekema, A.; Apperloo, R. C. \& de Lange, J. A rare complication during the surgical removal of an impacted maxillary third molar. Ned. Tijdschr. Tandheelkd.,119(7-8):363-6, 2012.

Kumar, A.; Srivastava, R. K.; Saxena, A.; Khanna, R. \& Ali, I. Removal of infected maxillary third molar from the infra-temporal fossa by caldwell luc procedure - Rare case report with literature review. J. Clin. Diagn. Res., 10(12):ZD01-ZD03, 2016.

Lutz, J. C.; Cazzato, R. L.; Le Roux, M. K. \& Bornert, F. Retrieving a displaced third molar from the infratemporal fossa: case report of a minimally invasive procedure. BMC Oral Health, 19(1):149, 2019.

Martínez, J. A. Cirugía Oral y Maxilofacial. Ciudad de México. El Manual Moderno, 2009. pp.263-85.

Netter, F. H. Atlas de Anatomía Humana. $4^{a}$ ed. Amsterdam, Elsevier, 2007. pp.34-6.

Nogueira, E. F. C.; Almeida, R. A. C.; Catunda, I. S; Vasconcelos, B. C. E. \& Leite Segundo, A. V. Impacted maxillary third molar displaced to the infratemporal space: a critical review and case report. Gen. Dent., 67(1):16-20, 2019.

Oberman, M.; Horowitz, I. \& Ramon, Y. Accidental displacement of impacted maxillary third molars. Int. J. Oral Maxillofac. Surg., 15(6):756-8, 1986

Orr 2nd, D. L. A technique for recovery of a third molar from the infratemporal fossa: case report. J. Oral Maxillofac. Surg., 57(12):1459-61, 1999.

Özer, N.; Üçem, F.; Saruhanog`lu, A.; Yilmaz, S. \& Tanyeri, H. Removal of a maxillary third molar displaced into pterygopalatine fossa via intraoral approach. Case Rep. Dent., 2013:392148, 2013

Polo, T. O. B.; Momesso, G. A. C.; de Lima, V. N.; Faverani, L. P.; Souza, F. Á. \& Garcia-Junior, I. R. Inappropriate management after accidental displacement of upper third molar to the infratemporal fossa may disrupt its subsequent removal. J. Craniofac. Surg., 28(3):e298-e299, 2017.

Primo, B. T.; Stringhini, D. J.; Klüppel, L. E.; Costa, D. J.; Rebellato, N. L. B. \& De Moraes, R. S. Delayed removal of maxillary third molar displaced into the infratemporal fossa. Rev. Esp. Cir. Oral Maxilofac., 36(2):78-81, 2014.
Roshanghias, K.; Peisker, A. \& Zieron, J. O. Maxillary tooth displacement in the infratemporal fossa. Dent. Res. J. (Isfahan), 13(4):373-5, 2016.

Selvi, F.; Cakarer, S.; Keskin, C. \& Ozyuvaci, H. Delayed removal of a maxillary third molar accidentally displaced into the infratemporal fossa. J. Craniofac. Surg., 22(4):1391-3, 2011.

Sencimen, M.; Gülses, A.; Secer, S.; Zerener, T. \& Özarslantürk, $S$. Delayed retrieval of a displaced maxillary third molar from infratemporal space via trans-sinusoidal approach: a case report and the review of the literature. Oral Maxillofac. Surg., 21(1):1-6, 2017.

Shahakbari, R.; Mortazavi, H. \& Eshghpour, M. First report of accidental displacement of mandibular third molar into infratemporal space. J. Oral Maxillofac. Surg., 69(5):1301-3, 2011.

Shruthi, T. M.; Shetty, A.; Imran, M.; Akash, K. S.; Ahmed, F. \& Ahmed, N. Removal of displaced maxillary third molar using modified Gillie's temporal approach. Ann. Maxillofac. Surg., 10(1):210-2, 2020.

Sverzut, C. E.; Trivellato, A. E.; Sverzut, A. T.; De Matos, F. P. \& Kato, R. B. Removal of a maxillary third molar accidentally displaced into the infratemporal fossa via intraoral approach under local anesthesia: report of a case. J. Oral Maxillofac. Surg., 67(6):1316-20, 2009.

Winkler, T.; von Wowern, N.; Odont, L. \& Bittmann, S. Retrieval of an upper third molar from the infratemporal space. J. Oral Surg., 35(2):130-2, 1977.

Yücesoy, T.; Ocak, H.; Er, N. \& Alkan, A. Removal of a supernumerary tooth displaced into the infratemporal fossa during extraction. Eur. Oral Res., 52(1):56-61, 2018.

Dirección para correspondencia:

Perla María Barrera Posada

Tecnológico de Monterrey

Escuela de Medicina y Ciencias de la Salud

Ave. Eugenio Garza Sada 2501

Monterrey, N.L.

México, 64849

MÉXICO

E-mail: perla.barrerap@gmail.com 